\title{
Reverse wave propagation in the cochlea
}

\author{
Wenxuan $\mathrm{He}^{\dagger \ddagger}$, Anders Fridberger ${ }^{\S}$ Edward Porsov ${ }^{\dagger}, \mathrm{Karl}_{\mathrm{G}}$ Grosh", and Tianying Ren ${ }^{\dagger \| t \dagger}$ \\ †Oregon Hearing Research Center, Department of Otolaryngology and Head and Neck Surgery, Oregon Health and Science University, 3181 Southwest Sam \\ Jackson Park Road, NRC04, Portland, OR 97239-3098; 'Department of Otolaryngology and Head and Neck Surgery of the First Affiliated Hospital, and \\ |Department of Physiology, Xi'an Jiaotong University, Xi'an, Shaanxi 710061, People's Republic of China; ${ }^{5}$ Center for Hearing and Communication \\ Research, Departments of Clinical Neuroscience and Otolaryngology, Karolinska Institutet, M1 Karolinska Universitetssjukhuset, SE-171 76 \\ Stockholm, Sweden; and "Departments of Mechanical Engineering and Biomedical Engineering, University of Michigan, Ann Arbor, \\ MI 48109
}

Edited by Charles D. Gilbert, The Rockefeller University, New York, NY, and approved December 31, 2007 (received for review August 27, 2007)

Otoacoustic emissions, sounds generated by the inner ear, are widely used for diagnosing hearing disorders and studying cochlear mechanics. However, it remains unclear how emissions travel from their generation sites to the cochlear base. The prevailing view is that emissions reach the cochlear base via a backward-traveling wave, a slow-propagating transverse wave, along the cochlear partition. A different view is that emissions propagate to the cochlear base via the cochlear fluids as a compressional wave, a fast longitudinal wave. These theories were experimentally tested in this study by measuring basilar membrane (BM) vibrations at the cubic distortion product (DP) frequency from two longitudinal locations with a laser interferometer. Generation sites of DPs were varied by changing frequencies of primary tones while keeping the frequency ratio constant. Here, we show that BM vibration amplitude and phase at the DP frequency are very similar to responses evoked by external tones. Importantly, the BM vibration phase at a basal location leads that at a more apical location, indicating a traveling wave that propagates in the forward direction. These data are in conflict with the backwardtraveling-wave theory but are consistent with the idea that the emission comes out of the cochlea predominantly through compressional waves in the cochlear fluids.

basilar membrane vibration | cochlear traveling wave laser interferometer | otoacoustic emission

K emp $(1,2)$ discovered that the cochlea can generate sounds that are transmitted to the external ear canal through the middle ear. Such otoacoustic emissions (OAEs) are considered a by-product of normal cochlear performance (3), relying on a putative cochlear amplifier (4-6). Because OAEs can provide information on the health status of the cochlea and can be noninvasively measured, they are increasingly used for diagnosing auditory disorders and as a research tool for studying cochlear physiology (3). However, the applications of OAEs have been limited because the generation and transmission mechanisms are not clear.

There are two different theories about OAE propagation from the generation site to the cochlear base. Propagation may either be dominated by a traveling wave moving in the backward direction $(1,7-9)$ or occur through a compressional wave in the cochlear fluids (10-16). Backward-traveling waves are slow and vibrate in the transverse direction, whereas compressional waves are longitudinal and travel at the speed of sound in water.

Backward-traveling waves were first postulated in Kemp's original reports $(1,2)$. Support for this theory comes from indirect measurements, primarily of the delay between the stimulus and the appearance of the emission in the ear canal (17). Although the interpretation of such measurements is not unambiguous, it is commonly believed that the backward delay is the same as the forward delay. Consequently, the emission delay would be twice the forward delay $(7,9,18)$. However, a direct proof of the theory requires backward waves to be measured from the basilar membrane (BM) at several different locations. The only such data, measured using a scanning laser interferom- eter, showed no evidence of backward-traveling waves (15). That study caused an intense debate on the mechanisms of reverse propagation of sound in the cochlea (19).

In this study, distortion products (DPs) were generated over a wide region of the cochlear length by using one of the most commonly used stimulation paradigms. Two tones, lowfrequency primary tone (f1) and high-frequency primary tone (f2) (f2 > f1) with constant f2/f1 ratio, were presented to the ear. A laser interferometer was used to measure cubic DPs (2f1-f2) from two BM longitudinal locations at different DP frequencies.

\section{Results}

Because of the invasive surgery for exposure of the BM and the vulnerability of sensitive hearing, the productivity of this experiment was very low. The data presented in Figs. 1-3 were collected from one sensitive cochlea, and those in Fig. 4 were from another sensitive preparation. Results from three additional sensitive animals were consistent with those presented below.

Fig. $1 A$ shows a direct comparison of the time waveform of DPs acquired from a basal location (blue line) and a more apical site (red line). The two longitudinal locations were separated by $196 \mu \mathrm{m}$. The best frequencies (BFs) of the two locations were $\approx 15$ and $\approx 12 \mathrm{kHz}$, respectively. A pair of tones at 9.00 and 9.45 $\mathrm{kHz}$ at 70 and $65 \mathrm{~dB}$ sound pressure level (SPL) was presented to produce DPs. To create Fig. $1 A$, the raw time domain data acquired from the $\mathrm{BM}$ were transformed to the frequency domain with the Fourier transform and the components at $\mathrm{f} 1$ and $\mathrm{f} 2$ were removed by editing the spectrum. Time domain waveforms containing spectral information at the DP frequencies were then generated by the inverse Fourier transform. In Fig. $1 A$, the major DP $(2 \mathrm{f} 1-\mathrm{f} 2=8.55 \mathrm{kHz})$ and primary frequencies (f1 $=9.00 \mathrm{kHz} ; \mathrm{f} 2=9.45 \mathrm{kHz}$ ) are below the BFs of the recording sites $(>12 \mathrm{kHz})$. This experimental design ensures that the measurement was made from locations (blue and red marks in Fig. $1 D$ ) basal to the DP generation site (green mark in Fig. $1 D$; the estimated spatial separation between the apical measured location and the $\mathrm{f} 2 \mathrm{BF}$ site is $\approx 550 \mu \mathrm{m})$. Under this condition, a backward-traveling wave would be evident at the measured locations. The basal response (Fig. $1 A$, blue line) is significantly smaller than the apical one (Fig. $1 A$, red line), which is also true for primary tone responses at these two locations. It is evident that the peaks of the blue curve in Fig. $1 A$ occur earlier than the red waveform in Fig. $1 A$, indicating a forward- rather than a backward-traveling wave. Cross-correlation analysis in Fig. $1 B$ shows that the basal location vibrated $\approx 20 \mu$ s earlier than the more apical site. In Fig. $1 C$, the delays from the basal to the more apical location (circles) are plotted as a function of the DP

Author contributions: W.H., E.P., and T.R. designed research; W.H. performed research; W.H., A.F., E.P., K.G., and T.R. analyzed data; and T.R. wrote the paper

The authors declare no conflict of interest.

This article is a PNAS Direct Submission.

${ }^{\dagger+}$ To whom correspondence should be addressed. E-mail: rent@ohsu.edu.

C 2008 by The National Academy of Sciences of the USA 

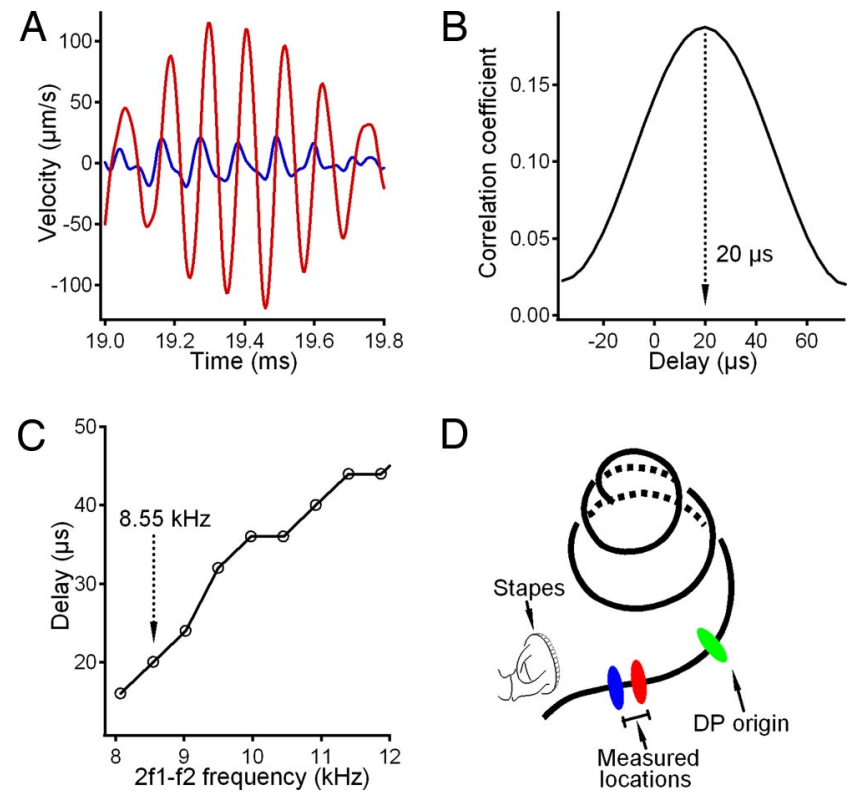

Fig. 1. The time relationship of BM vibrations. (A) The basal DP (blue line) occurs earlier than the more apical vibration (red line). (B) Cross-correlation analysis reveals an $\approx 20$ - $\mu$ s delay from the basal site to the more apical location. (C)The delay between the two locations increased with the DP frequency. $(D)$ Measurements were from longitudinal locations (blue and red) basal to the DP generation site (green), where the backward-traveling wave should dominate, if it exists.

frequency. The delay increased with frequency. Only data $<12$ $\mathrm{kHz}$ are presented in Fig. $1 C$ because backward-traveling waves are expected only in this frequency range. The arrow in Fig. $1 C$ indicates a $20-\mu$ s delay at $8.55 \mathrm{kHz}$, where the data in Fig. $1 A$ and $B$ were obtained. Thus, Fig. 1 shows that the DP propagated from the base toward the apex, as conventional traveling waves evoked by external sound. An alternative way of determining the direction of BM wave propagation is to examine the phase at the DP frequencies, as detailed in Fig. 2.

The magnitude and phase of the BM vibration at the DP frequency are presented in Fig. $2 A-D$. Data in Fig. $2 A$ and $B$ were collected from the more apical location, and those in Fig. $2 C$ and $D$ were measured from the basal location. The response at the DP frequency starts growing rapidly at $\approx 8 \mathrm{kHz}$, reaching a peak at the BFs of the observed locations (indicated by solid arrows in Fig. $2 A$ and $C$ ). The magnitude decreases quickly at higher frequencies. The overall shape of the magnitudefrequency functions of the DPs in Fig. $2 A$ and $C$ is similar to those of BM responses to single tone stimuli in Fig. $3 A$ and $C$, which is specifically shown by the black (DP) and red (tone responses) curves in Fig. $2 A$ and $C$. Although the tone response extends slightly more toward the low-frequency side, the DP and tone responses are very similar.

The phase of DP responses in Fig. $2 B$ and $D$ is presented with respect to the stapes vibration phase. Reference phase at the stapes $\left(\phi_{S D P}\right)$ was obtained from the f1 and $\mathrm{f} 2$ phase $\left(\phi_{s f 1}\right.$ and $\phi_{s f 2}$ ) according to $\phi_{s D P}=2 \phi_{s f 1}-\phi_{s f 2}$. The phase plotted in black in Fig. $2 B$ and $D$ was obtained by subtracting $\phi_{S D P}$ from the measured BM DP phase. The overall patterns of the phasefrequency relations at the two beads (in Fig. $2 B$ and $D$ ) are similar. The DP phase lag increases with increasing frequency. According to the backward-traveling theory, the backward wave should be detected only when the DP generation location is apical to the measured location (see Fig. $5 B$ ). DP frequencies $\left(F_{\text {DP }}\right)$ at the basal $(\approx 13.6 \mathrm{kHz})$ and more apical $(\approx 10.9 \mathrm{kHz})$ locations were calculated based on the $\mathrm{BF}$ at each location $\left(F_{\mathrm{BF}}\right)$
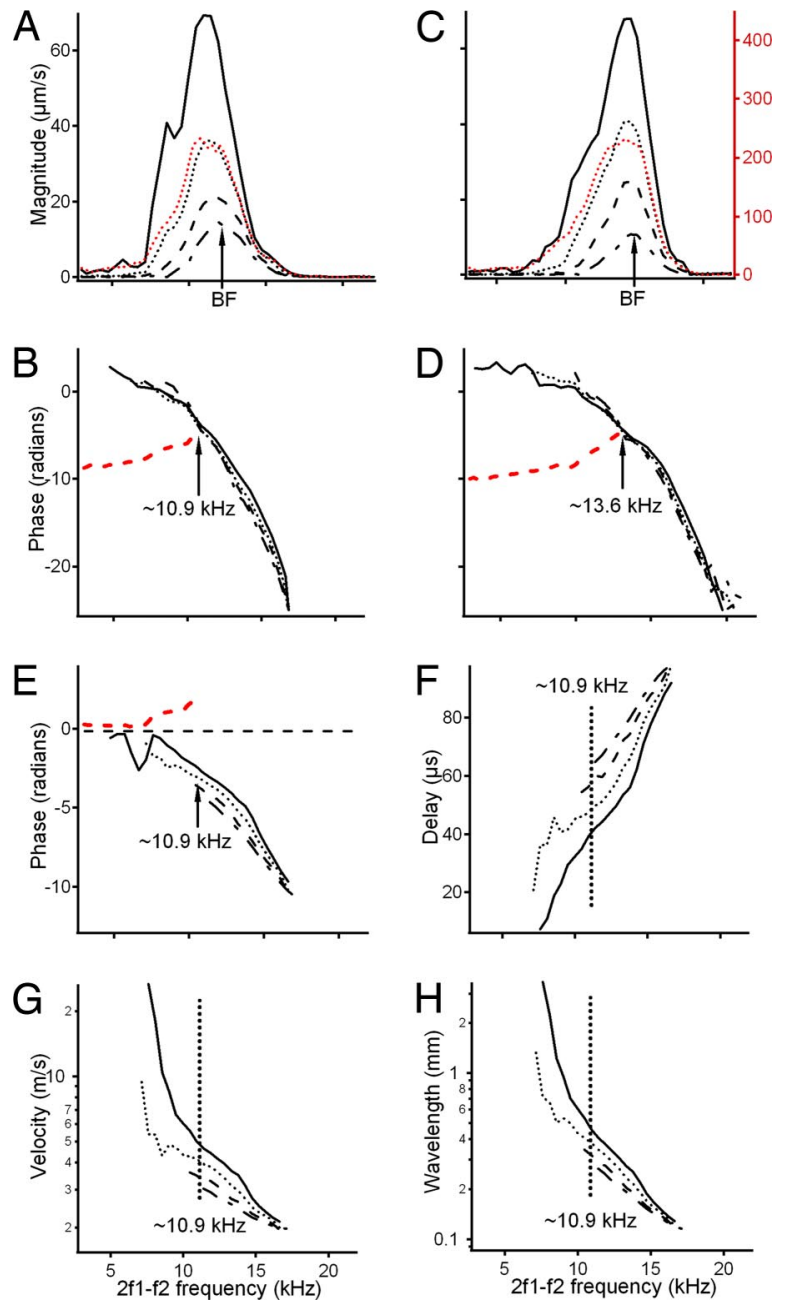

Fig. 2. Magnitude and phase of $B M$ vibrations at the DP frequency (primary levels of 40,50,60, and $70 \mathrm{~dB}$ SPL indicated by dash-dot, dashed, dotted, and solid black lines, respectively). These responses are very similar to BM responses to tones and show significant phase lags. $(A-E)$ The red dotted lines in $A$ and $C$ are BM responses to single tones at $60 \mathrm{~dB}$ SPL (scale on the right). Phase differences in $E$ show that, as the tone response in Fig. 3E, DP phase at the basal location leads the more apical phase. Red dashed lines in $B, D$, and $E$ show the predicted backward-traveling-wave phase, which are inconsistent with the data. $(F-H)$ The delay $(F)$, velocity $(G)$, and wavelength $(H)$ of the BM vibration at the DP frequency. $\mathrm{f} 2 / \mathrm{f} 1$ ratio, 1.05.

and the f2/f1 ratio $(R)$, i.e., $F_{\mathrm{DP}}=2^{*} F_{\mathrm{BF}} / R-F_{\mathrm{BF}}$. Thus, the DP phase at frequencies $<10.9 \mathrm{kHz}$ for the more apical location and $13.6 \mathrm{kHz}$ for more basal location should reveal the hypothetical backward-traveling wave. The hypothetical phases (shown by the red dashed lines in Fig. $2 B$ and $D$ ) were obtained by inverting the forward-traveling wave phases measured from the same locations. Evidently, the measured phase data are inconsistent with the phase) expected from the backward- traveling-wave theory (Fig. $2 B$ and $D$, red dashed lines). Instead, the DP phase data are similar to those of BM responses to external tones (Fig. $3 B$ and $D$ ). However, phase data from a single location (Fig. 2 $B$ or $D$ ) do not provide sufficient information for determining the propagation direction of the wave. To determine the wave direction, the phase difference of two locations (Fig. 2E) was obtained by subtracting the basal phase (Fig. $2 D$ ) from the more apical phase (Fig. 2B). Fig. $2 E$ shows that the basal location vibrates earlier than the more apical location, indicating that the wave propagates from base to apex. The waves propagate in the same direction at all frequencies and levels tested here. Although 

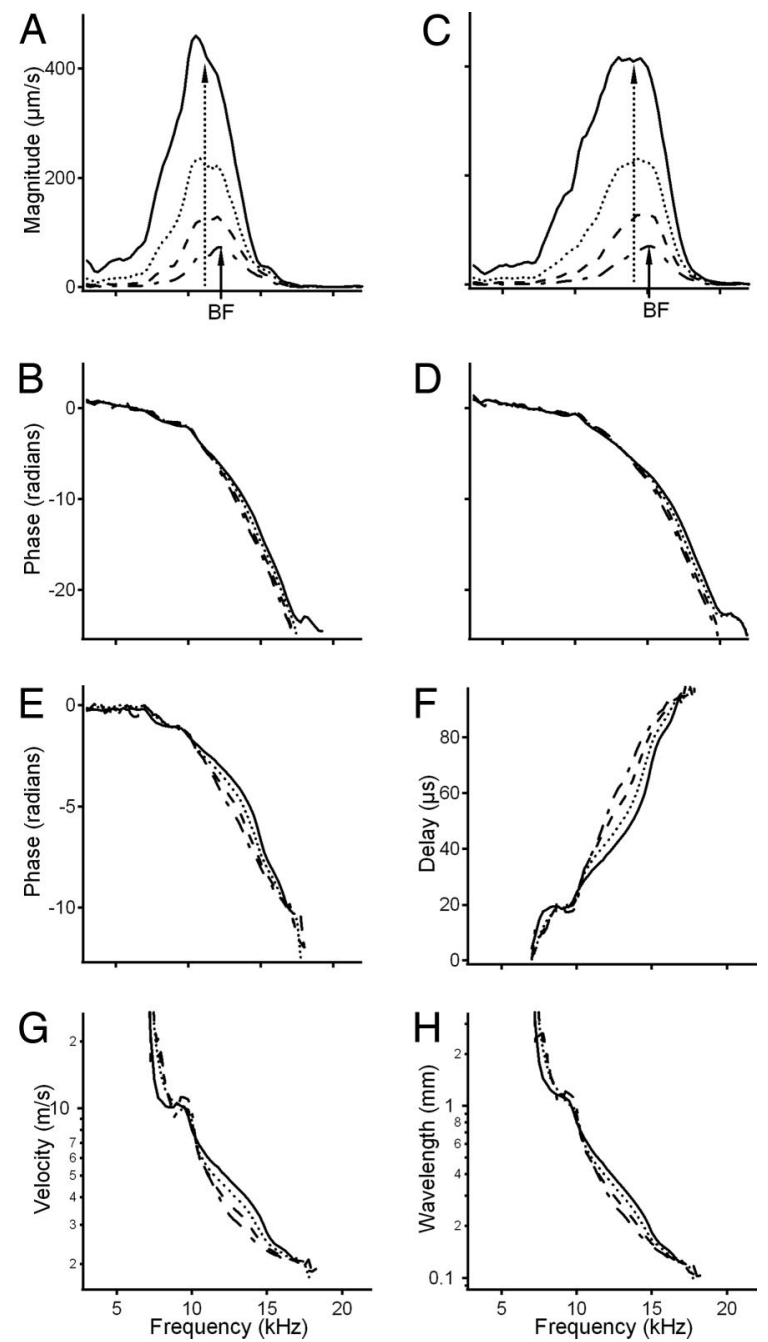

Fig. 3. Magnitude and phase responses of the BM to tones at different SPLs. Data were collected from the same two longitudinal locations as for Fig. 2 $(A-D)$ Similar tuning and phase patterns as in Fig. $2 A-D$ are shown. $(E)$ Phase difference shows that the basal phase leads the more apical phase at all levels. $(F-H)$ The delay $(F)$, velocity $(G)$, and wavelength $(H)$ are comparable to these in Fig. $2 \mathrm{~F}-\mathrm{H}$.

phase data becomes less reliable in the low-frequency range because of the small magnitude of the vibration, the phase difference $<8 \mathrm{kHz}$ (solid line in Fig. $2 E$ ) remains negative, consistent with a forward-traveling wave. The red curve in Fig. $2 E$ shows the predicted phase difference according to the backward-traveling-wave theory, which was obtained by inverting the phase curve at $70 \mathrm{~dB}$ SPL from Fig. $3 E$.

The delay between the two locations was calculated from the phase difference in Fig. $2 E$. The delay increases with frequency and decreases with intensity (Fig. $2 F$ ). The group velocity derived from the delay and the distance between the two locations is presented in Fig. 2G. Opposite to the delay, the group velocity decreases with the frequency and increases with the intensity. If the backward-traveling wave dominates the BM vibration at the DP frequency, delays and velocities at frequencies $<10.9 \mathrm{kHz}$ (the left side of the vertical dotted line in Fig. 2 $F$ and $G$ ) should be negative. Fig. $2 H$ shows that the wavelength decreases with frequency and increases with SPL. The wavelength $(\lambda)$ was obtained from the group velocity $(V)$ and frequency $(f): \lambda=V / f$.

To evaluate the similarity between the DP and tone responses, $\mathrm{BM}$ vibrations evoked by external tones were measured from the same two locations as for Fig. 2. Magnitude and phase responses to tones at 40 (dash-dot lines), 50 (dashed lines), 60 (dotted lines), and 70 (solid lines) dB SPL are presented in Fig. $3 A-D$. Shapes of the magnitude responses in Fig. $3 A$ and $C$ are similar to those in Fig. $2 A$ and $C$, which show similar peak frequencies. With intensity increase, in addition to the increase in magnitude, the response peak shifts from the original location (solid arrows) toward the low-frequency side (dotted arrows) in Fig. $3 A$ and $C$. This shift is not clearly visible in Fig. $2 A$ and $C$, likely because the levels are relatively low.

Phase responses to tones are presented in Fig. $3 B$ and $D$. Phase lag increases with frequency increase. The rate of lag increase is small at frequencies below the $\mathrm{BF}$ and large above the $\mathrm{BF}$. Although phase shows no change with intensity at frequencies below the BF, it shows slightly less lag at high stimulus level at frequencies above the BF. The phase difference between the two longitudinal locations, obtained by subtracting the basal phase (Fig. 3D) from the apical phase (Fig. 3B), is presented in Fig. $3 E$. The phase-frequency curve is approximately flat $<10$ $\mathrm{kHz}$ and becomes steep above this frequency. The delay between two locations, the group velocity, and the wavelength were calculated from Fig. $3 E$ and are presented in Fig. $3 F, G$, and $H$, respectively. Although shapes in Fig. $3 E-G$ are different from those in Fig. $2 E-G$, the ranges of the delay, velocity, and wavelength of tone responses are comparable to those of $\mathrm{BM}$ DP. A fundamental consistency between Figs. 2 and 3 is that both datasets show features of forward-traveling waves.

To ascertain whether the findings in Fig. 2 depend on the frequency separation between the primary tones, data were also collected with f2/f1 ratios of 1.10 and 1.20 (neither shown). Allowing minor across-animal differences, these data confirmed that BM DP is similar to the response evoked by external tones, and the phase difference between two longitudinal locations revealed a forward-traveling wave.

Because of the longitudinal extents of $\mathrm{f} 1$ and $\mathrm{f} 2$ traveling waves (20), it is possible that the observed DP response was generated locally at the measured location rather than being transmitted from the remote location near the $\mathrm{f} 2$ place. To explore this possibility, the relationship between DP and primary tone responses is presented in Fig. 4. Data were collected from a single location in the basal turn of a sensitive cochlea at $60 \mathrm{~dB}$ SPL f1 level and with f2/f1 ratios of 1.05, 1.10, and 1.20. Amplitudes and phases of f1 (Fig. 4, solid lines), f2 (Fig. 4, dotted lines), and 2f1-f2 (Fig. 4, thick solid lines) are plotted against DP frequencies (2f1-f2). The magnitude of the f1, f2, and $2 \mathrm{f} 1-\mathrm{f} 2$ all show peak responses near the BF of the observed location $(\approx 12$ $\mathrm{kHz}$ ). At $2 \mathrm{f} 1-\mathrm{f} 2$ frequencies $>12 \mathrm{kHz}$ (Fig. $4 E$ ), the $2 \mathrm{f} 1-\mathrm{f} 2$ magnitude can be greater than the $\mathrm{f} 1$ and $\mathrm{f} 2$ (Fig. $4 E$ Inset). The frequency location with maximum DP response is not the same as the maximal $\mathrm{f} 1$ or $\mathrm{f} 2$ responses. In addition, the DP phase $\left(\phi_{D P}\right)$ was calculated from the f1 and f2 phase $\left(\phi_{f 1}\right.$ and $\left.\phi_{f 2}\right)$, according to $\phi_{D P}=2 \phi_{f 1}-\phi_{f 2}$, and compared with measured DP phase. The measured DP phase (thick solid lines in Fig. $4 B, D$, and $F$ ) is clearly different from the DP phase predicted from the phase of the primaries (thick dotted lines in Fig. $4 B, D$, and $F$ ). This difference increases with the f2/f1 ratio.

\section{Discussion}

For result interpretation, the experimental design of the current study is presented in Fig. 5. The f1 tone-evoked traveling wave overlaps with that of $\mathrm{f} 2$, and $2 \mathrm{f} 1-\mathrm{f} 2 \mathrm{DP}$ is generated at this overlapped place (solid lines in Fig. 5A). According the backwardtraveling-wave theory $(1,7-9)$, the DP propagates in both directions: the forward wave reaches the DP frequency location and the backward wave reaches the stapes and gives rise to an emission in the ear canal. Because of the slow propagation of BM vibrations, both waves accumulate significant phase lags (delays) as they travel away from the DP generation site (solid lines in Fig. $5 B$ ). In the 

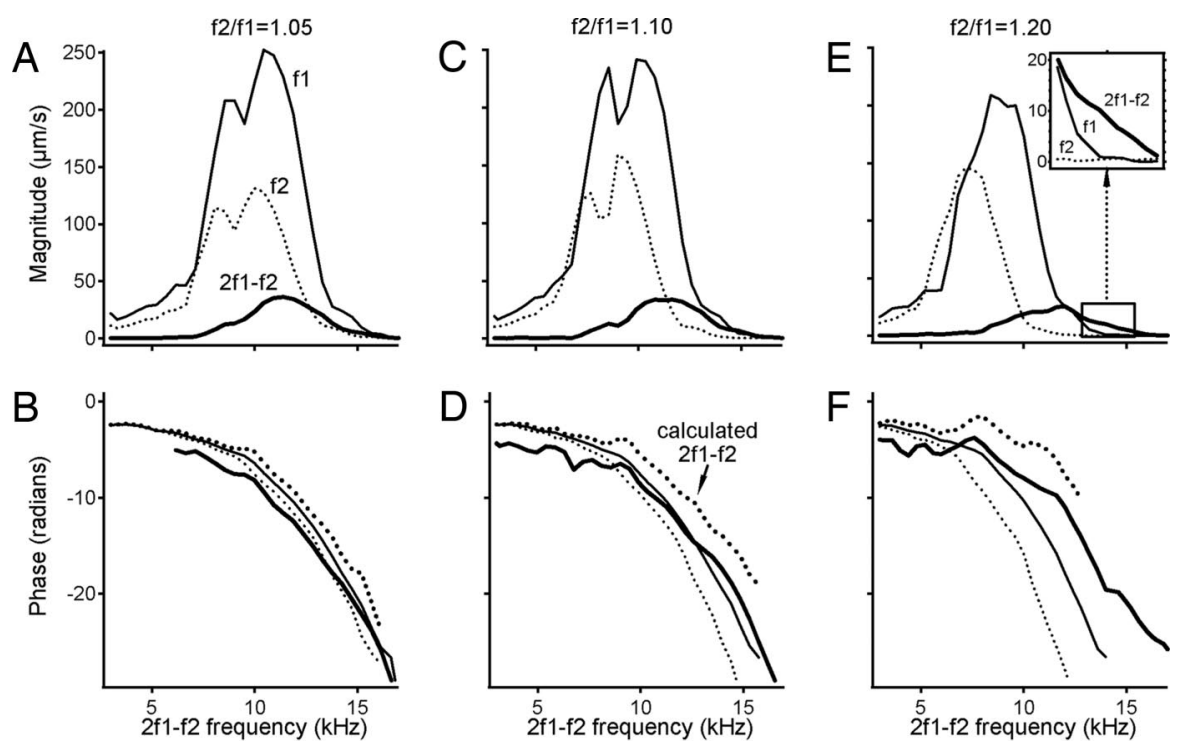

Fig. 4. Magnitude and phase relationship between primary tones and DP at $2 f 1-f 2$. Data are from a different animal than in Figs. 2 and 3 , at $60 \mathrm{~dB} S P L f 1$ intensity, and with $\mathrm{f} 2 / \mathrm{f} 1$ ratios of $1.05(A$ and $B), 1.10(C$ and $D)$, and 1.20 ( $E$ and $F$ ). Amplitudes and phases of $\mathrm{f} 1$ (thin solid lines), $\mathrm{f} 2$ (dotted lines), and $2 \mathrm{f} 1-\mathrm{f} 2$ (thick solid lines) are plotted against DP frequencies of $2 \mathrm{f} 1-\mathrm{f} 2$. Although $\mathrm{f1}, \mathrm{f2}$, and $2 \mathrm{f1}-\mathrm{f2}$ all show peak responses at a frequency near the $\mathrm{BF}(\approx 12 \mathrm{kHz})$, they reach the peaks at different $2 \mathrm{f} 1-\mathrm{f} 2$ frequencies. ( $E$ Inset) The $2 \mathrm{f} 1-\mathrm{f} 2$ can be significantly greater than the $\mathrm{f} 1$ or $\mathrm{f} 2$. In addition, the $2 \mathrm{f} 1-\mathrm{f} 2 \mathrm{phase}$ calculated from the $\mathrm{f} 1$ and $\mathrm{f} 2$ phases (thick dotted line) is different from the measured 2f1-f2 phase (thick solid line). This difference increases with the $\mathrm{f} 2 / \mathrm{f} 1 \mathrm{ratio}$.

compressional wave theory, the DP reaches the stapes through the cochlear fluids as a longitudinal wave (10-16). Because of the high speed, this reverse propagation does not introduce significant phase lag. Because DP wave exerts pressure on both oval and round windows, impedance mismatch between two windows creates a pressure difference across the BM and initiates a forward-traveling wave (21). This forward wave propagates to the DP frequency place (solid line in Fig. 5C). As the DP generation site is moved from the apex (solid lines in Fig. $5 C$ ) to the base (dotted lines in Fig. $5 C$ ) through the two observed locations (A and B), the backwardtraveling-wave theory predicts that DP phase at locations A and B should initially increase and then decrease, with a peak at $\mathrm{f} 2$ near the $\mathrm{BF}$ of the measured locations. Over the backward wave range, the DP phase at location A should be greater than that at location B. Compression-wave theory predicts that DP phase decreases with the frequency increase at both locations and the phase at location A should always lag the phase at location B. The data in Fig. 1 show that the DP reaches the basal location earlier than the more apical location. Fig. $2 B$ and $D$ shows no initial phase increase with $2 \mathrm{f} 1-\mathrm{f} 2$ frequency, and the DP phase at the basal location leads the phase at the more apical location. Phase difference between two locations (Fig. $2 E$ ) shows a delay from the basal to more apical location. These features are all consistent with a conventional forwardpropagating wave $(22,23)$ (Fig. $3 E$ ). One may argue that the backward wave may be so small that it cannot be detected with the current method. Then the question of why the backward wave is not the dominant vibration component at locations well basal to the primary regions needs to be answered.

The main critique against the fast wave hypothesis is that the results could be explained without invoking a fast reverse wave if the measured DP was locally generated (19). This concern was addressed by moving the DP generation site far from the measured location, toward the apex, and by comparing the phase relationship between measured DP and local f1 and f2. Although the BM tuning restricts the low-frequency responses (Fig. $2 A$ and $C$ ), the data in Fig. $2 B$ and $D$ reveal a phase decrease as a function of frequency at frequencies as low as $5 \mathrm{kHz}$. According to the frequency-location map of the gerbil cochlea (24), the BF location of $5 \mathrm{kHz}$ is $\approx 5.9 \mathrm{~mm}$ from the cochlear base. The measured locations are 3.0-3.9 $\mathrm{mm}$ from the base. Because of the separation between the DP generation site and the measured locations, it is unlikely that the observed DP was dominated by local components. In addition, if the measured DP is generated locally, the measured DP phase should have been consistent with the DP phase predicted, based on local f1 and f2 phase (19), and the DP magnitude should be proportional to local f1 and f2 magnitudes. The data in Fig. 4, however, show that the 2f1-f2 phase calculated from the local f1 and f 2 phase (thick dotted line) is different from the measured DP phase (thick solid line), and the DP peak frequency location is not consistent with those of primary tones $\mathrm{f} 1$ and $\mathrm{f} 2$ amplitude. These data indicate that the observed DP was not generated locally.

DP amplitude and phase responses are influenced by stimulus parameters (25), cochlear conditions (26), measured locations (27), and cochlear tuning (28). These undefined variables make the interpretation of BM data measured from a single location difficult. The use of two-location measurements dramatically simplified data interpretation, as the above factors contributed approximately equally to responses at the two locations. Twolocation phase data are of particular importance for determining the wave direction, because single-location data only quantifies group delay and provides no information on how the delay occurs. In addition, the use of reflective beads decreased the measurement noise floor by $\approx 20 \mathrm{~dB}$ to $\approx 0.1 \mu \mathrm{m} / \mathrm{s}$ in comparison to the scanning measurement (20). Thus, data from this study provide unambiguous information on wave propagation direction along the cochlear partition.

The experimental data in this study support the proposal that the backward propagation of the DP is dominated by fast waves and conflict with the commonly held backward-traveling-wave hypothesis. This finding is consistent with experimental results by von Bekesy (22), suggesting that pressure differences across the organ of Corti results in transverse waves that travel from base to apex irrespective of the source position within the cochlea. Zwislocki (21) theoretically explained this then so-called "paradox" in 1953. Now, the present experiments show that in the sensitive cochlea wave propagation in the forward direction dominates, irrespective of the source position (16). Although the conclusion of this study 


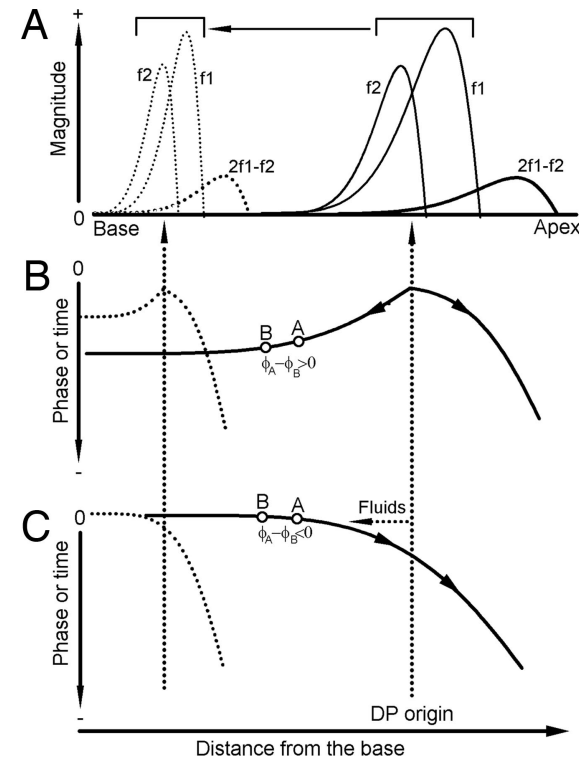

Fig. 5. Diagrams illustrating the experimental design and interpretation of the results. (A) 2f1-f2 DP is generated at the $\mathrm{f} 1$ and $\mathrm{f} 2$ traveling wave overlap place (solid lines). (B) The backward-traveling-wave theory predicts significant phase lags as the waves travel away from the DP generation site (solid lines). (C) In the cochlear compression wave mechanism, the DP reaches the stapes instantly through cochlear fluids as a longitudinal wave, which cannot be detected from the BM. However, the DP pressure in the cochlear fluid initiates a forward wave with significant phase lag (solid line). As the DP-generation site is moved from the apical region (solid line) to the base (dotted line) through locations A and B, the backward-traveling-wave theory predicts that DP phase at these locations would increase initially and then decrease, with a peak at $\mathrm{f} 2$ near the $\mathrm{BF}$ of the measured location. The apical phase leads the basal phase when $\mathrm{f} 2<\mathrm{BF}$. Compression-wave theory predicates that the DP phase decreases with the frequency at both locations, and the phase at the more apical location should always lag the phase at the basal location.

may not directly apply to other emissions, the backward-traveling wave generated hypothetically by a "reflection" mechanism (7) in stimulus frequency emissions has been excluded by the finding that the emission delay is approximately equal to or shorter than the forward delay (14).

\section{Experimental Procedures}

Young Mongolian gerbils (40-80 g; $n=30$ ) were used in this study. Animal protocols were the same as in previous studies $(15,20,29)$. Anesthesia was induced by i.p. injection of ketamine $(30 \mathrm{mg} / \mathrm{kg})$ followed by intramuscular xylazine ( $5 \mathrm{mg} / \mathrm{kg})$. The animal's head was fixed in a holder with $\mathrm{x}-\mathrm{y}$-z translation and rotation capability. A tracheotomy was performed to maintain free natural breathing. Body temperature was maintained at $38 \pm 1^{\circ} \mathrm{C}$ with a servo-regulated heating blanket. Animal procedures were approved by the Oregon Health and Science University Institutional Animal Care and Use Committee.

1. Kemp DT (1978) J Acoust Soc Am 64:1386-1391.

2. Kemp DT (1986) Hear Res 22:95-104.

3. Probst R, Lonsbury-Martin BL, Martin GK (1991) J Acoust Soc Am 89:2027-2067.

4. Davis H (1983) Hear Res 9:79-90.

5. Hudspeth A (1997) Curr Opin Neurobiol 7:480-486.

6. Dallos P (1992) J Neurosci 12:4575-4585

7. Shera CA, Guinan JJ, Jr (1999) J Acoust Soc Am 105:782-798.

8. Knight RD, Kemp DT (2001) J Acoust Soc Am 109:1513-1525.

9. Tubis A, Talmadge CL, Tong C (2000) J Acoust Soc Am 107:2112-2127.

10. Wilson JP (1980) Hear Res 2:527-532.

11. Robles L, Ruggero MA, Rich NC (1997) J Neurophysiol 77:2385-2399.

12. Avan P, Magnan P, Smurzynski J, Probst R, Dancer A (1998) Eur J Neurosci 10:1764-1770.

13. Ruggero MA (2004) Acoust Res Lett Online 5:143-147.

14. Siegel JH, Cerka AJ, Recio-Spinoso A, Temchin AN, van Dijk P, Ruggero MA (2005) J Acoust Soc Am 118:2434-2443.

15. Ren T (2004) Nat Neurosci 7:333-334.

16. Vetesnik A, Nobili R, Gummer A (2006) ORL J Otorhinolaryngol Relat Spec 68:347-352.
The left auditory bulla was exposed through a ventrolateral approach, and the round window membrane was removed. To expose the BM, the round window was enlarged by removing the bony edge with a miniature blade. After a clear view of $\approx 1 \mathrm{~mm}$ along the BM was achieved, a few gold-coated glass beads $(\approx 20$ $\mu \mathrm{m} \varnothing$ ) were dropped on the BM at locations with BFs from 12 to $22 \mathrm{kHz}$. To maintain its low impedance while eliminating optical distortions, the round window was partially covered with a thin glass coverslip. Cochlear sensitivity was monitored by measuring the compound action potential (20).

The technique for measuring BM vibration was similar to a previous study (20). The object beam of a heterodyne laser interferometer (OFV 302; Polytec) was focused on a bead on the BM through a long working-distance objective. The interferometer's output was proportional to the velocity of the bead along the optical axis. Beads have been shown to accurately follow the BM vibration (30). In good preparations, the noise floor of the measurement was $\approx 0.1 \mu \mathrm{m} / \mathrm{s}$. The BF of the observed location was determined as the frequency with the maximum amplitude in the magnitude transfer function at $40 \mathrm{~dB}$ SPL ( $0 \mathrm{~dB}$ SPL $=20 \mu \mathrm{Pa}$; the magnitude transfer function is the ratio of $\mathrm{BM}$ and stapes velocities as a function of frequency).

A custom program controlled the signal generation and data acquisition hardware (System II; Tucker-Davis Technologies). Simultaneous 23- or 43-ms tone bursts at $\mathrm{f} 1$ and $\mathrm{f} 2$ were generated by a D/A converter ( $1 \mathrm{~ms}$ rise/fall time). The signals were fed to a dual-channel power amplifier through two programmable attenuators and used to drive two earphones (ER-2; Etymotic Research). A sensitive microphone (10 B+; Etymotic Research) measured the sound pressure in the ear canal. The microphone-earphone probe was coupled into the ear canal to form a closed sound field. Signals from the microphone and the interferometer were digitized and averaged 10-40 times. The magnitude and phase of the averaged signal were obtained through the Fourier transform.

Vibrations at two longitudinal locations and the stapes footplate were measured as a function of the $f 1, f 2$, and $2 f 1-f 2$ frequencies. For experiments with $\mathrm{f} 2 / \mathrm{f} 1$ ratio of 1.05 , the $2 \mathrm{f1} 1 \mathrm{f} 2$ frequency was varied from 475 to $23,750 \mathrm{~Hz}$ in $475-\mathrm{Hz}$ steps by changing $\mathrm{f} 1$ and $\mathrm{f} 2$ simultaneously. For $\mathrm{f} 2 / \mathrm{f} 1$ ratios of 1.1 and 1.2 , the DP frequency was changed in steps of 450 and $400 \mathrm{~Hz}$, respectively. Data were collected at $\mathrm{f} 1$ intensities of $40,50,60$, and $70 \mathrm{~dB}$ SPL. The intensity of $\mathrm{f} 2$ was $5 \mathrm{~dB}$ below the $\mathrm{f} 1$ level. In the text, the stimulus intensity is presented by $\mathrm{f} 1$ level only.

The propagation direction of the DP wave was determined from the phase relationship between two BM locations. The wave propagates from a location with a phase lead to the location with a phase lag. The phase of the BM vibration was measured as a function of the DP frequency from the two locations. Phase difference $\left(\phi_{A-B}\right)$ was obtained by subtracting the basal phase $\left(\phi_{B}\right)$ from the apical phase $\left(\phi_{A}\right) ; \phi_{A-B}>0$ indicates backward-traveling waves, whereas $\phi_{A-B}<0$ is consistent with forward-traveling waves (27).

The delay from the basal to the more apical location $(t)$ was calculated from the phase difference $\left(\phi_{A-B}\right)$ and the frequency $(f)$ according to $t=\left(-\phi_{A-B}\right) /$ $(2 \pi f)$, where $t$ is in $\mathrm{S}, \phi_{A-B}$ is in radians, and $f$ is in $\mathrm{Hz}$. The group velocity of the $B M$ vibration ( $V$ ) was quantified according to $V=d / t$, where $d$ is the distance between two measured longitudinal locations, and $t$ is the delay over $d$. The distance $d$ was obtained from the $x, y$, and $z$ coordinates at locations $A$ and $B$, which were measured by using a positioning system consisting of a controller (ESP300) and three motorized linear translation stages (MFN25CC; Newport). The wavelength of the BM wave $(\lambda)$ was derived from the group velocity $(V)$ and frequency $(f): \lambda=V / f$, where $\lambda$ is in $\mathrm{m}, V$ is in $\mathrm{m} / \mathrm{s}$, and $f$ is in $\mathrm{Hz}$.

ACKNOWLEDGMENTS. We thank A. L. Nuttall for valuable discussions and three anonymous reviewers for suggestions and constructive comments. This work was supported by National Institute of Deafness and Other Communication Disorders Grants R01DC4554 (to T.R.) and R01DC4084 (to K.G.) and Swedish Research Council Grant K2005-04R-15371-01A (to A.F.).

17. Kimberley BP, Brown DK, Eggermont JJ (1993) J Acoust Soc Am 94:1343-1350.

18. de Boer E, Nuttall AL, Shera CA (2007) J Acoust Soc Am 121:352-362.

19. Shera CA, Tubis A, Talmadge CL (2006) in Auditory Mechanisms: Processes and Models, eds Nuttall AL, Ren T, Gillespie P, Grosh K, de Boer E (World Scientific, Singapore), pp 449-457. 20. Ren T (2002) Proc Natl Acad Sci USA 99:17101-17106.

21. Zwislocki JJ (1953) J Acoust Soc Am 25:986-989.

22. von Bekesy G (1960) Experiments in Hearing (McGraw-Hill, New York).

23. Zwislocki JJ (2002) Auditory Sound Transmission: An Autobiographical Perspective (Lawrence Erlbaum Associates, Mahwah, NJ).

24. Greenwood DD (1990) J Acoust Soc Am 87:2592-2605.

25. Tubis A, Talmadge CL, Tong C, Dhar S (2000) J Acoust Soc Am 108:1772-1785.

26. Mills DM, Rubel EW (1994) Hear Res 77:183-199.

27. He W, Nuttall AL, Ren T (2007) Hear Res 228:112-122.

28. Dong W, Olson ES (2005) J Acoust Soc Am 117:2999-3015.

29. Ren T, He W, Scott M, Nuttall AL (2006) J Neurophysiol 96:2785-2791.

30. Cooper NP (1999) J Acoust Soc Am 106:L59-L64. 